\title{
Mechanisms underlying the impact of long-chain $n-3$ PUFA on vascular reactivity
}

\author{
Wachira Jirarattanarangsri, Jeremy Spencer and Anne Marie Minihane \\ University of Reading, Reading, UK
}

CVD is the leading cause of death in the world. About eighteen million individuals worldwide die from the disease annually. Mortality rates vary considerably between countries; rates are lower in Japan and Mediterranean countries and highest in Eastern Europe ${ }^{(1)}$. Vascular function and reactivity is emerging as an important CVD risk factor and there is accumulating evidence to suggest that the amount and composition of fat in the diet is a major factor influencing endothelial cell function and vascular tone. Many studies have shown that dietary $n$-3 PUFA may have beneficial effects on vascular reactivity ${ }^{(2)}$. The endothelial NO synthase (eNOS) enzyme plays an important role in the regulation of vascular function, as it produces NO, which is an important vasodilator. However, the impact of longchain $n$-3 PUFA on eNOS activity remains poorly understood. The aim of the present in vitro study was to determine the impact of the long-chain $n$-3 PUFA EPA, docosapentaenoic acid (DPA) and DHA on NO production and eNOS regulatory pathways in human umbilical vein endothelial cells (HUVEC).

HUVEC (70-90\% confluence) were incubated for $30 \mathrm{~min}, 4 \mathrm{~h}$ and $24 \mathrm{~h}$ with the individual fatty acids in BSA complex solution $(2.5: 1$, $\mathrm{v} / \mathrm{v})$ at $0-100 \mu \mathrm{M}$. Cytotoxicity effects were measured by Lactate Dehydrogenase (LDH) assay and MTT assay. The LDH was quantified using the CytoTOX-ONE ${ }^{\mathrm{TM}}$ Assay from Promega UK Ltd. The MTT assay was adapted ${ }^{(3)}$. eNOS phosphorylation and eNOS concentration were analysed by Westernblotting techniques with the appropriate antibody. All experiments were repeated at least in triplicate.

There was no significant toxic effect of any of fatty acid concentration in either the LDH assay or the MTT assay. The present study demonstrated that fish oil fatty acids (DHA, EPA and DPA) and Palmitic acid (0-100 $\mu \mathrm{M}$ for $30 \mathrm{~min}, 4$ and $24 \mathrm{~h})$ influenced eNOS phosphorylation and eNOS protein level in HUVEC. There was no significant effect on the ratio of eNOS phosphorylation and eNOS concentration for most of the fatty acids except with the $24 \mathrm{hr}$ incubation time at $0,1,10$ and $100 \mu \mathrm{M}$ DHA $(P \leq 0.04)$ and with the $4 \mathrm{hr}$ incubation at 25 and $100 \mu \mathrm{M}$ PA $(P \leq 0.01)$. There was a trend toward increased eNOS phosphorylation to concentration ratio at 4 hrs in response to EPA with a dose effect evident but this did not reach significance $(P>0.05)$ (Figure 1). This result indicated that an increase in eNOS phosphorylation and eNOS protein level with EPA treatment may positively induce eNOS activity and NO production. In conclusion, there was little evidence of an effect of EPA, DPA or DHA on eNOS phosphorylation status in HUVEC cells, with evidence of a decrease following $24 \mathrm{~h}$ of exposure to DHA.

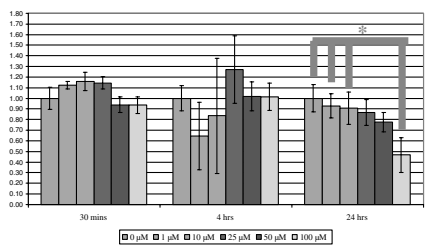

(a)

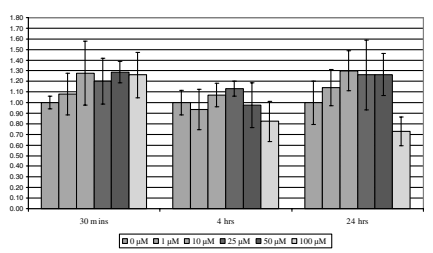

(b)

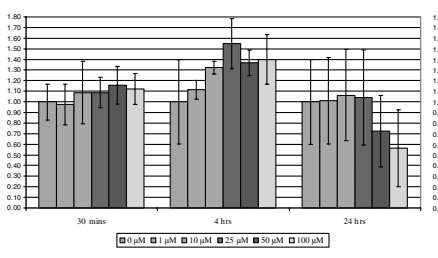

(c)

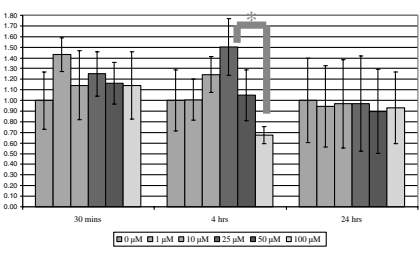

(d)

Figure 1. Phospho ser 1177 eNOS density: eNOS density ratio. HUVECs incubated with individual fatty acids (a) Docosahexaenoic acid (DHA) (b) Docosapentaenoic acid (DPA) (c) Eicosapeintaenoic acid (EPA) and (d) Palmitic acid. Data was presented as relative to control. Values are presented as means with SEM indicated. $*$ indicated significant concentration dependent effects $P \leq 0.04$ for DHA and $P \leq 0.01$ for PA.

1. World Health Organization (2007) Cardiovascular disease: prevention and control. http://www.who.int/dietphysicalactivity/publications/facts/cvd/en/

2. Hall W, Sanders K, Sanders T \& Chowienczyk P (2008) The Journal of Nutrtion 138, 287-291.

3. Michaelis M, Michaelis R, Fleming I, Suhan T, Cinatl J, Blaheta R, Hoffmann K, Kotchetkov R, Busse R, Nau H \& Cinatl J (2004) Mol Pharmacol 65, $520-527$. 